\title{
Edge-to-edge repair: past challenge, current case selection and future advances
}

\author{
Paul Sorajja, Anene C. Ukaigwe \\ Center for Valve and Structural Heart Disease, Minneapolis Heart Institute Foundation, Abbott Northwestern Hospital, Minneapolis, MN, USA \\ Correspondence to: Paul Sorajja, MD. Roger L. and Lynn C. Headrick Family Chair, Director, Valve Science Center, Minneapolis Heart Institute \\ Foundation, Abbott Northwestern Hospital, 920 East 28th Street, Minneapolis, MN 55407, USA. Email: paul.sorajja@allina.com.
}

\begin{abstract}
Severe mitral regurgitation (MR) is associated with heart failure and impaired survival with an annual mortality risk in excess of $5 \%$ per year for unoperated patients. Despite availability of surgical mitral valve interventions, as many as half of all patients with severe MR do not receive interventions. Transcatheter edge-to-edge repair with MitraClip has been a revolutionary therapy for MR, with over 100,000 treated patients worldwide. The usage has also expanded to different challenging anatomies as well as tricuspid regurgitation. Additionally, other transcatheter edge-to-edge repair devices are being studied. The evolution of these devices as well as what to expect in the future will be discussed here.
\end{abstract}

Keywords: Mitral repair; transcatheter; regurgitation

Submitted Apr 21, 2020. Accepted for publication Jul 27, 2020.

doi: 10.21037/acs-2020-mv-17

View this article at: http://dx.doi.org/10.21037/acs-2020-mv-17

\section{Introduction}

Despite the availability of life-saving surgical mitral valve therapies, up to $50 \%$ of mitral regurgitation (MR) patients do not receive interventions $(1,2)$. Transcatheter edgeto-edge repair has revolutionized the treatment of such patients with symptomatic severe MR. Percutaneously performing an Alfieri stitch was first done in 2003. The MitraClip repair system (Abbott Vascular, USA) received Conformité Européenne (CE) mark in 2008 and Food and Drug Administration (FDA) approval in 2013. More recently, a new device PASCAL (Edwards Lifesciences, USA) has received CE mark for treatment of MR in Europe. Over 100,000 patients have been treated with edgeto-edge catheter-based therapy, and the understanding of the indications and outcomes are now elementary to the management of patients with MR.

\section{Classification of MR}

MR should be classified according to etiology. Primary MR results from intrinsic mitral valve abnormalities due to myxomatous degeneration or fibroelastic deficiency causing flail or prolapse leaflets [i.e., degenerative MR (DMR)], but can also be rheumatic, infectious, radiation-induced, among other causes. Secondary or functional mitral regurgitation (FMR) results from changes in the left ventricle (LV) due to dilation or ischemia, which causes MR from annular dilation, leaflet tethering and/or restriction.

\section{Device design}

The MitraClip system comprises of a steerable guide catheter (SGC) and a clip delivery system (CDS) on which the clips of varying sizes is mounted. The clip is made of polyester covered cobalt chromium arms and a nitinol gripper line. The initial clip design, the NTR, was $5 \mathrm{~mm}$ wide in the grasping area. The clip measured $17 \mathrm{~mm}$ with arms opening to 120 degrees. This was followed by XTR clips with longer clip arms measuring $22 \mathrm{~mm}$ with arms opening to 120 degrees. Recently, a fourth generation of clips "G4" consisting of four different clip sizes (NT, XT, NTW, XTW) were released. The NT and XT clips are $4 \mathrm{~mm}$ wide in the grasping area while the NTW and XTW are $6 \mathrm{~mm}$ wide in the grasping area. The G4 clips also features controlled grasping actuation 
enabling simultaneous or sequential leaflet grasping and optimization of leaflet grasping. These extra features provide a wider variety of clips to be tailored to patient's anatomy and allow previously challenging mitral valve anatomy to be treated with this technology. Continuous left atrial pressure monitoring as an adjunct to transesophageal echocardiographic assessment of results is also enabled with this fourth generation of clips.

\section{Transcatheter edge-to-edge repair studies}

The Endovascular Valve Edge-to-edge Repair Trial (EVEREST II trial) was the pivotal U.S. trial, which enrolled 279 patients with predominantly DMR (3). They were randomized 2:1 to receive percutaneous repair with a MitraClip $v s$. conventional surgery. Freedom from death, recurrent $M R$, and repeat interventions was higher in the surgical arm ( $73 \%$ vs. $55 \%)$. Residual MR needing mitral valve surgery was higher in the MitraClip arm (20\% vs. $2 \%)$. MitraClip was however safer than surgery at thirty days. Major adverse events at thirty days occurred in $15 \%$ of the MitraClip group compared to $48 \%$ in the surgical group, with the main driver of the difference being greater blood transfusions in the surgery patients. Only $2 \%$ of percutaneous repair patients were NYHA III-IV compared to $13 \%$ of surgical patients at thirty days. The relatively inferior MR reduction with MitraClip was notable, and potentially explained by early use of first generation technology.

At five years, the primary efficacy endpoint of the trial (i.e., freedom from death, mitral valve surgery, reintervention, and moderate to severe MR) was $44 \% v s$. $64 \%$ in the MitraClip and surgery arms respectively. This is driven primarily by higher residual MR and need for reintervention in the MitraClip group within the first year. Importantly the acute results of MitraClip were durable with few repeat interventions required in follow-up. At five years, there was no difference in the mortality and functional class between the two groups. Between one and five years, there was no significant difference in the two groups with respect to need for repeat interventions (4).

The EVEREST II High Risk Registry and REALISM (Real world Expanded Multicenter Study of the MitraClip System) Continued Access Study High-Risk Arm prospectively enrolled patients with Society of Thoracic Surgery Predicted Risk of Mortality (STS PROM) score of $12 \%$ or greater (5). In the 351 patients enrolled, MR reduction to moderate or less was achieved in $86 \%$, which was associated with improved functional status and reduced heart failure hospitalizations. Similar findings were demonstrated in a two-phase observational study of the MitraClip ${ }^{\circledR}$ System in Europe (ACCESS-EU) (6). The procedural successes noted in these studies likely reflect the increased experience with the expanded use of the MitraClip system.

While MitraClip was used predominantly for FMR patients in Europe after CE-Mark approval, this was pursued without randomized data supporting clinical benefit. As a result, several pivotal trials of MitraClip for patients with FMR were undertaken.

MITRA-FR (Multicentre Study of Percutaneous Mitral Valve Repair MitraClip Device in Patients with Severe Secondary Mitral Regurgitation) is a trial that randomized 307 patients with FMR and severe LV dysfunction in France to MitraClip with medical therapy vs. medical therapy alone. There was no difference in mortality (HR 1.11; 95\% CI, 0.69-1.77; $\mathrm{P}=0.66$ ) or the composite of death and unplanned heart failure hospitalization (HR 1.16; $95 \%$ CI, 0.73-1.84; $\mathrm{P}=0.66$ ) between the two groups (7).

The COAPT trial (Cardiovascular Outcomes Assessment of the MitraClip Percutaneous Therapy for Heart Failure Patients with FMR) randomized 614 patients with heart failure and moderate to severe and severe FMR to maximally tolerated guideline directed medical therapy (GDMT) alone or GDMT with MitraClip. Patients with severe pulmonary hypertension and right ventricular failure were excluded. This trial showed a profound benefit of MitraClip at two years consisting of reduced mortality and heart failure hospitalization (HR 0.57; 95\% CI, 0.44-0.71; $\mathrm{P}<0.001$ ) and all-cause mortality (HR 0.62; 95\% CI, 0.46$0.82 ; \mathrm{P}<0.001)(8)$.

There are various explanations for the widely different outcomes of the two trials of MitraClip in patients with FMR. Maximally tolerated GDMT was required in the COAPT trial but was site-managed and less aggressive in MITRA-FR. More residual MR was seen in the MITRAFR study (17\% vs. $5 \%)$ at one year compared to COAPT. The severity of MR was less in MITRA-FR (baseline EROA $<0.3 \mathrm{~cm}^{2}$ in $52 \%$ ) compared to COAPT (EROA $\geq 0.4 \mathrm{~cm}^{2}$ in $\left.41 \%\right)$. MITRA-FR enrolled patients with larger LV diastolic volumes $(252 \pm 67 \mathrm{cc})$ than COAPT $(192 \pm 67 \mathrm{cc})$. These observations have led to a theoretical concept of disproportionate MR in FMR as another explanation for the discrepancies in both trials. Patients with disproportionate MR (i.e., MR that is more than can be explained by $L V$ enlargement alone) may benefit relatively more from mitral 
valve therapies in contrast to those with proportionate MR. This concept is yet to be prospectively evaluated $(9,10)$.

The Randomized study of the MitraClip device in Heart Failure patients with clinically significant FMR (RESHAPE -HF2) trial (NCT 02444338) (11) is also evaluating MitraClip therapy for FMR, and may offer some additional insight into the role of this therapy for FMR patients.

In the CLASP (The CLASP Study Edwards PASCAL TrAnScatheter Mitral Valve RePair System Study) Early Feasibility study, 62 patients with severe MR refractory to medical therapy were treated with the PASCAL device (Edwards Lifesciences). Fifty-six percent had FMR and $8 \%$ had mixed MR. MR reduction was attained in $98 \%$ of patients, with all-cause mortality of $1.6 \%$. Major adverse events was $6.5 \%$ (12). The PASCAL system received CE mark 2019. In the U.S., The CLASP IID/F (NCT 03706833) is currently enrolling MR patients and randomizing them to 2:1 PASCAL or MitraClip (13).

\section{Case selection}

\section{Access}

The procedure is typically performed via transfemoral and transseptal access, although transjugular access has also been reported.

The ideal location of transseptal puncture to maximize chances of a successful procedure are $4.0-4.5 \mathrm{~cm}$ from the mitral annular plane, or $4.5-5.0 \mathrm{~cm}$ from the level of coaptation. This can be modified based on the mitral valve pathology. For instance, greater heights are acceptable for flail leaflets since the pathologies extend above the mitral annular plane, while slightly lower transseptal heights may be accepted to treat secondary MR, and, in some instances, pathology that is primarily located laterally.

\section{Clip location}

MitraClip deployment between the middle scallops of the anterior and posterior leaflets at the A2/P2 location was mandated in the EVEREST II trial, and is currently associated with less residual MR and the lowest need for repeat intervention. Meta-analysis of single center observational studies comparing outcomes of MitraClip with A2/P2 pathology and other EVEREST II trial criteria vs. MitraClip in patients not meeting these criteria have shown higher risk of recurrent MR in FMR and complex mitral valve etiology with flail. Initial MitraClip experience from the Society of Thoracic Surgeons (STS) and Transcatheter Valve Therapies (TVT) registry showed that MitraClip in A2/P2 location was associated with greater $M R$ reduction, less residual $M R$, less conversion to surgery and lower in hospital mortality (14). In this study, the majority of patients had DMR making generalization less applicable to FMR.

In real world experience, MitraClip can be safely placed between the lateral scallops at A1/P1 or medial scallops A3/ P3 close to the commissures to reduce MR due to flail or prolapse in these locations. MitraClip in these areas has the least impact on mitral gradients post MitraClip. Given that these are located in the chords, care must be taken when manipulating MitraClips in these areas to avoid injury and worsening MR.

\section{Large flail segments}

Large flail width $\geq 15 \mathrm{~mm}$ and flail gap $\geq 10 \mathrm{~mm}$ historically have been associated with increased need for re-intervention after MitraClip therapy (15). While these patients were excluded from the EVEREST, new generation technology (NTR and XTR) and new techniques (e.g., adenosine, rapid pacing and positive end expiratory pressure) enable successful therapy of these pathologies. Although there was a signal for increased leaflet damage causing single leaflet device attachment with new generation technology, implementation of guidelines for device selection and operator experience is associated with lower risk of injury (16). The G4 system, with controlled gripper actuation, also facilitates independent grasping of leaflets which would otherwise be challenging with MitraClip. A post market study of the G4 system is underway in the EXPAND and EXPAND G4 studies respectively $(17,18)$.

\section{Degree of MR and residual MR}

The degree of MR was shown in the TVT registry to directly impact successful MR reduction. The greater the MR and the presence of multiple jets negatively impacts outcomes of MitraClip as $\geq 2$ residual MR as well as need for $>1$ clip may be associated with increased gradients.

Single center observational studies demonstrate that degree of residual MR after MitraClip is a predictor of long term outcomes (19). Similar findings were demonstrated in analysis of the COAPT trial. The EVOLVE MR trial (MitraClip for the Treatment of Moderate FMR, NCT 03705312) (20) will evaluate the benefits, if any, of 
MitraClip therapy in moderate FMR.

Residual MR after MitraClip can pose a challenge for further MitraClip therapy due to elevated mean mitral gradients. Transcatheter methods for addressing these have been described with Amplatzer Vascular Plugs (Abbott Vascular, Santa Clara USA) (21).

\section{Mitral valve area (MVA) and mean mitral gradient}

The greatest reduction in MVA post MitraClip occurs with $\mathrm{A} 2 / \mathrm{P} 2$ placement due to significant reduction in mitral valve septal-lateral diameter. Clips placed closer to the commissures have relatively less impact on MVA and gradients. The TVT registry showed that $20 \%$ of MitraClips were performed in patients with MVA $\leq 4 \mathrm{~cm}^{2}$, mean gradient $>4 \mathrm{mmHg}$ or mitral annular calcification without negatively impacting outcomes (14). This implies that MitraClip is possible in carefully selected patients with these characteristics. However, elevated mean mitral gradient, mitral annular calcification and use of multiple clips are associated with high post procedural mitral gradients (22).

\section{Left ventricular ejection fraction and lv size}

Trials evaluating MitraClip repair excluded patients with left ventricular ejection fracture (LVEF) $<30 \%$, which is a reflection of the American and European guidelines for management of valve diseases that raise caution against mitral valve surgery in patients with severely reduced LV systolic function. In the TRAMI (transcatheter mitral valve interventions) registry which analyzed 823 patients treated with MitraClip, LVEF $<30 \%$ was identified as a predictor of one year mortality (23). More contemporary reports from the TRAMI registry identified no difference in safety, efficacy and clinical improvement in patients undergoing MitraClip therapy with LVEF $<30 \%$ or $>30 \%$. Procedural failure was a strong predictor of mortality in the low LVEF cohort. There have been reports of MitraClip therapy successfully used to bridge a patient to advanced heart failure therapies (24). This is yet to be tested in larger studies. The MITRABRIDGE registry (NCT04293575) has been designed to help give insights to MitraClip therapy in this situation (25).

\section{Operator experience}

Recent analysis of the STS database shows that there is a learning curve for MitraClip implantation with procedural outcomes and mortality improving with more experience irrespective of etiology of MR (26). Operators with greater experience, that is $>50$ cases, were more likely to treat functional MR, deploy clips at the commissures when needed, and use more than one clip per patient. The prevalence of complications was predictably lower in operators with greater case experience driven by cardiac injury and need for blood transfusions. Death, stroke or conversion to surgery was unaffected by operator experience.

Overall procedural success defined as moderate or less residual MR was high ( $>91.4 \%$ ) even in the least experienced operators and improved with increasing experience. The learning curve to obtain these results was $92 \%$ after 10 cases, $93 \%$ at 50 cases and $95 \%$ at 150 cases. Operators with increasing experience were more likely to have optimal residual MR of mild or less (64\% for operators 1-25 cases, $68 \%$ in operators $26-50$ cases and $75 \%$ in operators with $>50$ cases). The learning curve to obtain these optimal results are steeper corresponding to $65 \%$ after 10 cases, $73 \%$ after 50 cases and $80 \%$ after 150 cases. Operator case experience had no impact on post procedure mean gradient. There was a $33 \%$ reduction in operator case time between operators with $1-25$ cases and those with $>50$ cases.

\section{Special situations}

\section{Hypertrophic cardiomyopathy (HCM)}

Transcatheter repair with MitraClip has been successfully used in HCM and MR to plicate the mitral valve hence limiting the systolic anterior motion of the mitral valve and reducing left ventricular outflow tract (LVOT) gradients and MR (27). In early experience, this therapy led to improved LVOT gradients, left atrial pressure and NYHA functional status. Care must be taken to use the therapy in patients in whom MR is severe, and without small MVA. Due to the dynamic nature of MR in obstructive HCM, the mitral annulus and left atrium are frequently not enlarged.

\section{Previous surgical repair}

MitraClip has been used for recurrent MR in patients with previous annuloplasty ring repair (21). The anterior mitral leaflet may be clipped to the annuloplasty ring where the posterior leaflet has previously been resected. MitraClip repair in this situation may also be limited by often already 
elevated baseline gradients in these patients and small MVA due to down sized annuloplasty repair.

\section{Acute severe MR after myocardial infarction}

There have been case reports and case series of this being successfully performed in patients with papillary muscle rupture $(28,29)$.

MitraClip has successfully been used to alleviate symptoms and adverse hemodynamics in cardiogenic shock patients with severe MR (30,31). The acute hemodynamic effects of MitraClip in this cohort of patients resulted in decreased pulmonary artery, LV and atrial pressure resulting in increased cardiac output leading to recovery. Finally, MitraClip does not preclude further surgical therapies of surgical mitral valve or advanced heart failure therapies. This has not been systematically studied and predictors of outcomes as well as impact on survival is not clear.

\section{Future advances}

\section{Clip management strategies}

Previously, the presence of a MitraClip did not allow for transcatheter mitral valve replacement options. Given the rate of recurrent $M R$, it is desirable to have perform other transcatheter therapies where possible if additional MitraClip therapy is not feasible due to significant elevated mitral gradient.

Catheter based electrosurgical techniques have been employed to cut the anterior mitral tissue bridge and allow subsequent transcatheter mitral valve replacement (32). This complementary approach allows for transcatheter mitral valve replacement and elimination of MR in these high-risk patients.

\section{Other selection of clips}

Other technologies for transcatheter edge-to-edge repair are the PASCAL which is currently being evaluated in the USA as part of a clinical trial. CLASP IID/F trial (NCT 03706833) designed to evaluate the efficacy and safety of the PASCAL system compared to the MitraClip (13).

\section{Conclusions}

MitraClip therapy is a revolutionary treatment for MR.
Since its approval for DMR, MitraClip therapy has evolved to include treatment of other indications including diseases of the mitral and tricuspid valve. Clinical trials, registry data and innovative use as well as collaboration with industry is expected to lead to continued improvements in device design to simplify its use and expand the scope of indications.

\section{Acknowledgments}

Funding: None.

\section{Footnote}

Conflicts of Interest: PS: Consultant for Abbott Structural, Medtronic, Boston Scientific, Edwards Lifesciences, Admedus, and W.L. Gore; and has received institutional research grants from Abbott Structural, Medtronic, and Boston Scientific. ACU has no conflicts of interest to declare.

Open Access Statement: This is an Open Access article distributed in accordance with the Creative Commons Attribution-NonCommercial-NoDerivs 4.0 International License (CC BY-NC-ND 4.0), which permits the noncommercial replication and distribution of the article with the strict proviso that no changes or edits are made and the original work is properly cited (including links to both the formal publication through the relevant DOI and the license). See: https://creativecommons.org/licenses/by-nc-nd/4.0/.

\section{References}

1. Goel SS, Bajaj N, Aggarwal B, et al. Prevalence and outcomes of unoperated patients with severe symptomatic mitral regurgitation and heart failure: comprehensive analysis to determine the potential role of MitraClip for this unmet need. J Am Coll Cardiol 2014;63:185-6.

2. Iung B, Baron G, Butchart EG, et al. A prospective survey of patients with valvular heart disease in Europe: The Euro Heart Survey on Valvular Heart Disease. Eur Heart J 2003;24:1231-43.

3. Feldman T, Foster E, Glower DD, et al. Percutaneous repair or surgery for mitral regurgitation. $\mathrm{N}$ Engl J Med 2011;364:1395-406.

4. Kar S, Feldman T, Qasim A, et al. Five-year outcomes of transcatheter reduction of significant mitral regurgitation in high-surgical-risk patients. Heart 2019;105:1622-8. 
5. Glower DD, Kar S, Trento A, et al. Percutaneous mitral valve repair for mitral regurgitation in high-risk patients: results of the EVEREST II study. J Am Coll Cardiol 2014;64:172-81.

6. Maisano F, Franzen O, Baldus S, et al. Percutaneous mitral valve interventions in the real world: early and 1-year results from the ACCESS-EU, a prospective, multicenter, nonrandomized post-approval study of the MitraClip therapy in Europe. J Am Coll Cardiol 2013;62:1052-61.

7. Obadia JF, Messika-Zeitoun D, Leurent G, et al. Percutaneous Repair or Medical Treatment for Secondary Mitral Regurgitation. N Engl J Med 2018;379:2297-306.

8. Stone GW, Lindenfeld J, Abraham WT, et al. Transcatheter Mitral-Valve Repair in Patients with Heart Failure. N Engl J Med 2018;379:2307-18.

9. Grayburn PA, Sannino A, Packer M. Proportionate and Disproportionate Functional Mitral Regurgitation: A New Conceptual Framework That Reconciles the Results of the MITRA-FR and COAPT Trials. JACC Cardiovasc Imaging 2019;12:353-62.

10. Packer M, Grayburn PA. Contrasting Effects of Pharmacological, Procedural, and Surgical Interventions on Proportionate and Disproportionate Functional Mitral Regurgitation in Chronic Heart Failure. Circulation 2019;140:779-89.

11. Anon. RESHAPE-HF2. Accessed March 23, 2020. Available online: https://clinicaltrials.gov/ct2/show/ NCT02444338

12. Lim DS, Kar S, Spargias K, et al. Transcatheter Valve Repair for Patients With Mitral Regurgitation: 30-Day Results of the CLASP Study. JACC Cardiovasc Interv 2019;12:1369-78.

13. Anon. CLASP IID/IIF. Accessed March 20, 2020. Available online: https://clinicaltrials.gov/ct2/show/ NCT03706833

14. Sorajja P, Mack M, Vemulapalli S, et al. Initial Experience With Commercial Transcatheter Mitral Valve Repair in the United States. J Am Coll Cardiol 2016;67:1129-40.

15. Lesevic H, Karl M, Braun D, et al. Long-Term Outcomes After MitraClip Implantation According to the Presence or Absence of EVEREST Inclusion Criteria. Am J Cardiol 2017;119:1255-61.

16. Praz F, Braun D, Unterhuber M, et al. Edge-to-Edge Mitral Valve Repair With Extended Clip Arms: Early Experience From a Multicenter Observational Study. JACC Cardiovasc Interv 2019;12:1356-65.
17. Anon. MitraClip EXPAND Study. Accessed March 23, 2020. Available online: https://clinicaltrials.gov/ct2/show/ NCT03502811

18. Anon. EXPAND G4. Accessed March 23, 2020. Available online: https://clinicaltrials.gov/ct2/show/ NCT04177394

19. Orban M, Orban M, Lesevic H, et al. Predictors for longterm survival after transcatheter edge-to-edge mitral valve repair. J Interv Cardiol 2017;30:226-33.

20. Anon. EVOLVE MR. Accessed March 23, 2020. Available online: https://clinicaltrials.gov/ct2/show/ NCT03705312

21. Niikura H, Bae R, Gössl M, et al. Transcatheter Mitral Valve Repair of Recurrent Mitral Regurgitation Following Mitral Surgery. JACC Cardiovasc Interv 2019;12:1395-7.

22. Thaden JJ, Malouf JF, Nkomo VT, et al. Mitral Valve Anatomic Predictors of Hemodynamic Success With Transcatheter Mitral Valve Repair. J Am Heart Assoc 2018;7:e007315.

23. Puls M, Lubos E, Boekstegers P, et al. One-year outcomes and predictors of mortality after MitraClip therapy in contemporary clinical practice: results from the German transcatheter mitral valve interventions registry. Eur Heart J 2016;37:703-12.

24. Garatti A, Castelvecchio S, Bandera F, et al. Mitraclip procedure as a bridge therapy in a patient with heart failure listed for heart transplantation. Ann Thorac Surg 2015;99:1796-9.

25. Anon. MitraBridge Registry. Accessed March 23, 2020. Available online: https://clinicaltrials.gov/ct2/show/ NCT04293575

26. Chhatriwalla AK, Vemulapalli S, Szerlip M, et al. Operator Experience and Outcomes of Transcatheter Mitral Valve Repair in the United States. J Am Coll Cardiol 2019;74:2955-65.

27. Sorajja P, Pedersen WA, Bae R, et al. First Experience with Percutaneous Mitral Valve Plication as Primary Therapy for Symptomatic Obstructive Hypertrophic Cardiomyopathy. J Am Coll Cardiol 2016;67:2811-8.

28. Bilge $M$, Alemdar R, Yasar AS. Successful percutaneous mitral valve repair with the MitraClip system of acute mitral regurgitation due to papillary muscle rupture as complication of acute myocardial infarction. Catheter Cardiovasc Interv 2014;83:E137-40.

29. Goel SS, Zuck V, Nallamothu N, et al. Successful MitraClip for Severe Mitral Regurgitation Secondary to Papillary Muscle Rupture as a Complication of 
Transcatheter Aortic Valve Replacement. JACC

Cardiovasc Interv 2018;11:e47-8.

30. Adamo M, Curello S, Chiari E, et al. Percutaneous edgeto-edge mitral valve repair for the treatment of acute mitral regurgitation complicating myocardial infarction: A single centre experience. Int J Cardiol 2017;234:53-7.

31. Estevez-Loureiro R, Adamo M, Arzamendi D, et al. Transcatheter mitral valve repair in patients with acute

Cite this article as: Sorajja P, Ukaigwe AC. Edge-to-edge repair: past challenge, current case selection and future advances. Ann Cardiothorac Surg 2021;10(1):43-49. doi: 10.21037/acs-2020-mv-17 myocardial infarction: insights from the European Registry of MitraClip in Acute Mitral Regurgitation following an acute myocardial infarction (EREMMI). EuroIntervention 2020;15:1248-50.

32. Sorajja P, Bae R, Gössl M, et al. Complementary Transcatheter Therapy for Mitral Regurgitation. J Am Coll Cardiol 2019;73:1103-4. 